\title{
The canonical embedding of an unramified morphism in an étale morphism
}

\author{
David Rydh
}

Received: 1 October 2009 / Accepted: 30 January 2010 / Published online: 11 March 2010

(C) The Author(s) 2010. This article is published with open access at Springerlink.com

\begin{abstract}
We show that every unramified morphism $X \rightarrow Y$ has a canonical and universal factorization $X \hookrightarrow E_{X / Y} \rightarrow Y$ where the first morphism is a closed embedding and the second is étale (but not separated).
\end{abstract}

Keywords Unramified · étale $\cdot$ étale envelope $\cdot$ Stack

Mathematics Subject Classification (2000) Primary 14A20

\section{Introduction}

It is well-known that any unramified morphism $f: X \rightarrow Y$ of schemes (or Deligne-Mumford stacks) is an étale-local embedding, i.e., there exists a commutative diagram

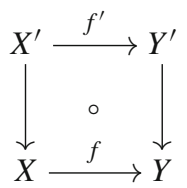

where $f^{\prime}$ is a closed embedding and the vertical morphisms are étale and surjective. To see this, take étale presentations $Y^{\prime} \rightarrow Y$ and $X^{\prime} \rightarrow X \times_{Y} Y^{\prime}$ such that $X^{\prime}$ and $Y^{\prime}$ are schemes and then apply [3, Cor. 18.4.7]. This proof utterly fails if $Y$ is a stack which is not DeligneMumford and the existence of a diagram (*) appears to be unknown in this case. Also, if we require $Y^{\prime} \rightarrow Y$ to be separated, then in general there is no canonical choice of the diagram $(*)$.

Supported by the Swedish Research Council.

D. Rydh ( $\varangle)$

Department of Mathematics, University of California, Berkeley,

970 Evans Hall \#3840, Berkeley, CA 94720-3840, USA

e-mail: dary@math.berkeley.edu 
The purpose of this article is to show that for an arbitrary unramified morphism of algebraic stacks, there is a canonical étale morphism $E_{X / Y} \rightarrow Y$ and a closed embedding $X \hookrightarrow E_{X / Y}$ over $Y$. If $f: X \rightarrow Y$ is an unramified morphism of schemes (or algebraic spaces), then $E_{X / Y}$ is an algebraic space.

Remark 1.1 If $f: X \rightarrow Y$ is an immersion, then there is a canonical factorization $X \hookrightarrow$ $U \rightarrow Y$ where $X \hookrightarrow U$ is a closed immersion and $U \rightarrow Y$ is an open immersion. Here $U$ is the largest open neighborhood of $X$ such that $X$ is closed in $U$. Explicitly, $U=Y \backslash(\bar{X} \backslash X)$. This factorization commutes with flat base change if $f$ is quasi-compact but not with arbitrary base change unless $f$ is a closed immersion. The canonical factorization that we will construct is slightly different and commutes with arbitrary base change but is not separated. For an immersion $f: X \rightarrow Y$, the scheme $E_{X / Y}$ is the gluing of $U$ and $Y$ along the open subsets $U \backslash X=Y \backslash \bar{X}$.

Theorem 1.2 Let $f: X \rightarrow Y$ be an unramified morphism of algebraic stacks. Then there exists an étale morphism $e=e_{f}: E_{X / Y} \rightarrow Y$ together with a closed immersion $i=$ $i_{f}: X \hookrightarrow E_{X / Y}$ and an open immersion $j=j_{f}: Y \rightarrow E_{X / Y}$ such that $f=e \circ i$, $\operatorname{id}_{Y}=e \circ j$ and the complement of $i(X)$ is $j(Y)$. We have that:

(i) The triple $(e, i, j)$ is unique up to unique 2-isomorphism, i.e., if $e^{\prime}: E^{\prime} \rightarrow Y$ is an étale morphism, $i^{\prime}: X \hookrightarrow E^{\prime}$ is a closed immersion and $j^{\prime}: Y \rightarrow E^{\prime}$ is an open immersion over $Y$ such that the complement of $i^{\prime}(X)$ is $j^{\prime}(Y)$, then there is an isomorphism $\varphi: E^{\prime} \rightarrow E_{X / Y}$ such that $e^{\prime}=e \circ \varphi, i=\varphi \circ i^{\prime}$ and $j=\varphi \circ j^{\prime}$, and $\varphi$ is unique up to unique 2-isomorphism.

(ii) Let $g: Y^{\prime} \rightarrow Y$ be any morphism and let $f^{\prime}: X^{\prime} \rightarrow Y^{\prime}$ be the pull-back of $f$ along $g$. Then the pull-backs of $e_{f}, i_{f}$ and $j_{f}$ along $g$ coincide with $e_{f^{\prime}}, i_{f^{\prime}}$ and $j_{f^{\prime}}$.

(iii) $e$ is an isomorphism if and only if $X=\emptyset$.

(iv) $e$ is separated if and only if $f$ is étale and separated.

(v) e is universally closed (resp. quasi-compact, resp. representable) if and only if $f$ is so. In particular, $e$ is universally closed, quasi-compact and representable if $f$ is finite.

(vi) $e$ is of finite presentation (resp. quasi-separated) if and only if $f$ is of constructible finite type (resp. quasi-separated and locally of constructible finite type). For the definition of the latter notions, see Appendix D.

(vii) $e$ is a local isomorphism if and only if $f$ is a local immersion.

(viii) If $g: V \rightarrow X$ is an étale morphism, then there exists a unique étale morphism $g_{*}: E_{V / Y} \rightarrow E_{X / Y}$ such that the pull-back of $i_{f}$ (resp. $j_{f}$ ) along $g_{*} i_{\text {s }} i_{f \circ g}$ (resp. $j_{f \circ g}$ ). If $g$ is surjective (resp. representable, resp. an open immersion), then so is $g_{*}$.

(ix) If $g: V \rightarrow X$ is a closed immersion then there is a natural surjective morphism $g^{*}: E_{X / Y} \rightarrow E_{V / Y}$ such that $i_{f \circ g}=g^{*} \circ i_{f} \circ g$ and $j_{f \circ g}=g^{*} \circ j_{f}$. The morphism $g^{*}$ is an isomorphism if and only if $g$ is a nil-immersion (i.e., a bijective closed immersion). If $g$ is an open and closed immersion, then $g^{*} g_{*}=\mathrm{id}_{E_{V / Y}}$.

We call the étale morphism $e: E_{X / Y} \rightarrow Y$ the étale envelope of $X \rightarrow Y$. Note that the fibers of $e$ coincide with the fibers of $X \amalg Y \rightarrow Y$. In Definition 3.1 (resp. 4.1) we give a functorial description of $E_{X / Y}$ in the representable (resp. general) case.

For the definitions of representable and unramified morphisms of stacks, see Appendices A and B. If the reader does not care about stacks, then rest assured that any scheme (or algebraic space) is an algebraic stack and that any morphism of schemes (or algebraic spaces) is representable. For schemes (or algebraic spaces), unique up to unique 2-isomorphism means unique up to unique isomorphism. 
Remark 1.3 Even if $f: X \rightarrow Y$ is a morphism of schemes (as is the case if $Y$ is a scheme and $f$ is representable and separated), it is often the case that $E_{X / Y}$ is not a scheme but an algebraic space, cf. Example 2.5. However, if $f$ is a local immersion, then $E_{X / Y}$ is a scheme by (vii).

Remark 1.4 For any representable morphism $f: X \rightarrow Y$ locally of finite type one can define a natural operation $f_{\#}$ : $\mathbf{S e t}_{*}(X) \rightarrow \operatorname{Set}_{*}(Y)$ on étale sheaves of pointed sets such that if $f$ is unramified, then the étale envelope $E_{X / Y}$ is the sheaf $f_{\#}\{0,1\}_{X}$. Here $\{0,1\}_{X}$ denotes the constant sheaf of a pointed set with two elements. If $f$ is étale, then $f_{\#}$ is left adjoint to the pull-back $f^{-1}$ of pointed sets and if $f$ is a monomorphism, then $f_{\#}=f_{\text {! }}$ is extension by zero. We do not develop the general theory of $f_{\#}$ in this article.

Remark 1.5 Note that "quasi-compact" is equivalent to "finite type" for unramified morphisms. When $Y$ is non-noetherian, the question of finite presentation (or equivalently of quasi-separatedness) of $E_{X / Y} \rightarrow Y$ is somewhat delicate, cf. Appendix D.

We begin with a few examples of the étale envelope in Sect. 2. The proof of Theorem 1.2 in the representable case is given in Sect. 3 and the general case is dealt with in Sect. 4. Some applications of the main theorem are outlined in Sect. 5. In Appendix A we give precise meanings to "algebraic space", "algebraic stack" and "representable". In Appendix B we define unramified and étale morphisms of stacks and establish their basic properties. Some limit results used in the non-noetherian case are given in Appendix C. Finally, in Appendix D we define the technical condition "of constructible finite type" which is only used to give a characterization of the unramified morphisms having a finitely presented étale envelope in the non-noetherian case.

Theorem 1.2 was inspired by a similar result recently obtained by Anca and Andrei Mustaţă [9]. They study the case when $f: X \rightarrow Y$ is a finite unramified morphism between proper integral noetherian Deligne-Mumford stacks and construct a stack $F_{X / Y}$ such that $F_{X / Y} \rightarrow Y$ is étale and universally closed and such that $F_{X / Y} \times_{Y} f(X)$ is a union of closed substacks $\left\{F_{i}\right\}$ which admit étale and universally closed morphisms $F_{i} \rightarrow X$. The stack $F_{X / Y}$ has an explicit groupoid description but a functorial interpretation is missing. In general, $F_{X / Y}$ is different from $E_{X / Y}$ and does not commute with arbitrary base change.

\section{Examples}

Example 2.1 If $f: X \rightarrow Y$ is étale. Then $E_{X / Y}=X \amalg Y$.

Example 2.2 Let $Y$ be a scheme and let $X=\coprod_{i=1}^{n} X_{i}$ be the disjoint union of closed subschemes $X_{i} \hookrightarrow Y$. Then $E_{X / Y}$ is a scheme and can be described as the gluing of $n+1$ copies of $Y$ as follows. Let $Y_{i}=Y$ for $i=1, \ldots, n$. Glue each $Y_{i}$ to $j(Y)=Y$ along $Y \backslash X_{i}$. The resulting scheme is $E_{X / Y}$. Note that $Y_{i} \cap Y_{j}=Y \backslash\left(X_{i} \cup X_{j}\right)$.

Example 2.3 The following example is a special case of the previous example. Let $Y=$ $\operatorname{Spec}(k[x, y] / x y)$ be the union of the two coordinate axes in the affine plane and let $X=$ $\mathbb{A}^{1} \amalg \mathbb{A}^{1}$ be the normalization of $Y$. Then $E_{X / Y}$ can be covered by three affine open subsets isomorphic to $Y$. If we denote these three subsets by $j(Y), Y_{1}, Y_{2}$, then $j(Y) \cap Y_{1}$ is the open subset $y \neq 0, j(Y) \cap Y_{2}$ is the open subset $x \neq 0$ and $Y_{1} \cap Y_{2}=\emptyset$.

Example 2.4 Let $Y$ be a nodal cubic curve in $\mathbb{P}^{2}$ and let $f: X \rightarrow Y$ be the normalization. Let $0 \in Y$ be the node and let $\{+1,-1\} \subseteq X$ be its preimage. The scheme $E_{X / Y}$ has two 
irreducible components $X$ and $\overline{j(Y)}$ and $\overline{j(Y)}$ is isomorphic to the gluing of $Y$ with $X$ along $Y \backslash\{0\}$ and $X \backslash\{+1,-1\}$. The scheme $E_{X / Y}$ is covered by two open separated subschemes $j(Y)$ and $U$. The open subset $U=X_{1} \cup X_{2}$ is the union of two copies of $X$, the first is $i(X)$ and the second is $\overline{j(Y)} \backslash\{0\}$, such that $\pm 1 \in X_{1}$ is identified with $\mp 1 \in X_{2}$. The intersection of $j(Y)$ and $U$ is $j(Y) \backslash 0=X_{2} \backslash\{+1,-1\}$.

Example 2.5 Let $Y$ be an irreducible scheme, $Z \hookrightarrow Y$ an irreducible closed subscheme, $Z \neq Y$, and $g: X \rightarrow Z$ a non-trivial étale double cover. Then $E_{X / Y}$ is an algebraic space which is not a scheme. In fact, let $E=E_{X / Y} \backslash j(Z)$. Then $E \subseteq E_{X / Y}$ is open and $\left.e\right|_{E}: E \rightarrow Y$ is universally closed and such that $\left.e\right|_{E}$ is an isomorphism outside $Z$ and coincides with $g$ over $Z$. If $\xi$ is the generic point of $Z$, then $E_{\xi}=\{\eta\}$ where $\eta$ is the generic point of $X$. If $E$ was a scheme, then $E \times_{Y} \operatorname{Spec}\left(\mathcal{O}_{Y, \xi}\right)$ would be a local scheme with closed point $\eta$ and in particular separated. This would imply that $E \times_{Y} \operatorname{Spec}\left(\mathcal{O}_{Y, \xi}\right) \rightarrow \operatorname{Spec}\left(\mathcal{O}_{Y, \xi}\right)$ is finite and étale. But $E \rightarrow Y$ has generic rank 1 and special rank 2 .

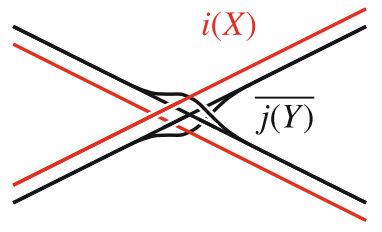

Example 2.3

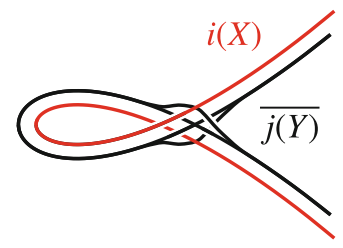

Example 2.4

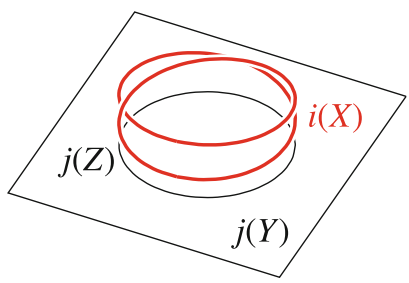

Example 2.5

\section{The representable case}

In this section we prove Theorem 1.2 for representable unramified morphisms.

Definition 3.1 Let $f: X \rightarrow Y$ be an unramified morphism of algebraic spaces. We define a contravariant functor $E_{X / Y}: \mathbf{S c h}_{/ Y} \rightarrow$ Set as follows. For any scheme $T$ and morphism $T \rightarrow Y$, we let $E_{X / Y}(T)$ be the set of commutative diagrams

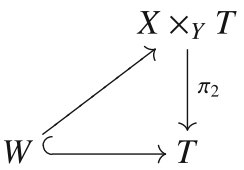

such that $W \rightarrow X \times{ }_{Y} T$ is an open immersion and $W \rightarrow T$ is a closed immersion. Pull-backs are defined by pulling back such diagrams.

The presheaf $E_{X / Y}$ is a presheaf of pointed sets. The distinguished element of $E_{X / Y}(T)$ is given by $W=\emptyset$. It is also naturally a presheaf in partially ordered sets and if $f$ is separated, then any two elements $W_{1}, W_{2} \in E_{X / Y}(T)$ have a greatest lower bound given by $W_{1} \cap W_{2}$.

By fpqc-descent of open subsets and of closed immersions, we have that $E_{X / Y}$ is a sheaf

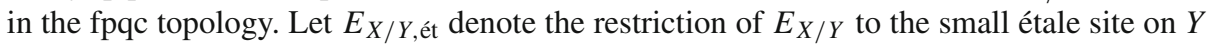
so that $E_{X / Y \text {,ét }}$ is an étale sheaf. The first goal is to show that $E_{X / Y}$ is locally constructible, i.e., that $E_{X / Y}$ is the extension of $E_{X / Y \text {,ét }}$ to the big étale site. 
Lemma 3.2 The functor $E_{X / Y}$ is locally of finite presentation, i.e., for every inverse limit of affine schemes $T=\lim _{\longleftarrow} T_{\lambda}$ over $Y$ we have that

$$
\underset{\lambda}{\lim _{\lambda}} E_{X / Y}\left(T_{\lambda}\right) \rightarrow E_{X / Y}(T)
$$

is bijective.

Proof An element of $E_{X / Y}(T)$ is an open immersion $w: W \rightarrow X \times_{Y} T$ such that $\pi_{2} \circ$ $w: W \rightarrow T$ is a closed immersion. As $w$ is locally of finite presentation and $W$ is affine, there is by Proposition C.1 an étale morphism $w_{\lambda}: W_{\lambda} \rightarrow X \times_{Y} T_{\lambda}$ such that $W_{\lambda}$ is quasicompact and quasi-separated and the pull-back of $w_{\lambda}$ along $T \rightarrow T_{\lambda}$ is $w$. After increasing $\lambda$ we can also assume that the morphism $\pi_{2} \circ w_{\lambda}: W_{\lambda} \rightarrow T_{\lambda}$ is a closed immersion by Proposition C.2. Then $w_{\lambda}$ is an étale monomorphism and hence an open immersion. The open immersion $w_{\lambda}$ determines an element of $E_{X / Y}\left(T_{\lambda}\right)$ which maps to $w$ so the map in the lemma is surjective.

That the map is injective follows immediately from [3, Thm. 8.8.2 (i)] since if $w_{\lambda}: W_{\lambda} \rightarrow$ $X \times_{Y} T_{\lambda}$ is an object of $E_{X / Y}\left(T_{\lambda}\right)$ then $W_{\lambda}$ is quasi-compact and quasi-separated and $w_{\lambda}$ is locally of finite presentation.

The following lemma is well-known for separated unramified morphisms.

Lemma 3.3 Let $S=\operatorname{Spec}(A)$ be the spectrum of a strictly henselian local ring with closed point $s$, let $X$ be an algebraic space and let $X \rightarrow S$ be an unramified morphism.

(i) Let $x: \operatorname{Spec}(k(s)) \rightarrow X_{s}$ be a point in the closed fiber. Then the henselian local scheme $X(x):=\operatorname{Spec}\left(\mathcal{O}_{X, x}\right)$ is an open subscheme of $X$ and $X(x) \rightarrow S$ is a closed immersion. In particular, $X=X_{1} \cup X_{2}$ is a union of open subspaces where $X_{1}$ is a scheme and $X_{2} \cap X_{s}=\emptyset$.

(ii) There is a one-to-one correspondence between points of $\left|X_{s}\right|$ and non-empty open subspaces $W \subseteq X$ such that $W \rightarrow S$ is a closed immersion. This correspondence takes $x \in\left|X_{S}\right|$ to $X(x) \subseteq X$ and $W \subseteq X$ to $W \cap\left|X_{S}\right|$.

Proof Let $V \rightarrow X$ be an étale presentation with $V$ a separated scheme and choose a lifting $v: \operatorname{Spec}(k(s)) \rightarrow V_{s}$ of $x$. Then $V_{1}=\operatorname{Spec}\left(\mathcal{O}_{V, v}\right) \cong X(x)$ is an open and closed neighborhood of $v$ and $V_{1} \rightarrow S$ is finite and hence a closed immersion. It follows that $X(x) \cong V_{1} \rightarrow X$ is an open immersion. The second statement follows immediately from the first.

Lemma 3.4 Let $f: X \rightarrow Y$ be an unramified morphism of algebraic spaces and let $\bar{y} \rightarrow Y$ be a geometric point. The stalk $\left(E_{X / Y, e ́ t}\right)_{\bar{y}}$ equals $\left|X_{\bar{y}}\right| \cup\{\emptyset\}$ where $\left|X_{\bar{y}}\right|$ is the underlying set of the geometric fiber $X_{\bar{y}}=X \times{ }_{Y} \operatorname{Spec}(k(\bar{y}))$.

Proof Let $Y(\bar{y})=\operatorname{Spec}\left(\mathcal{O}_{Y, \bar{y}}\right)$ denote the strict henselization of $Y$ at $\bar{y}$. We have that $\left(E_{X / Y \text {,ét }}\right)_{\bar{y}}=\lim _{\longrightarrow} E_{X / Y}(U)$ where the limit is over all étale neighborhoods $U \rightarrow Y$ of

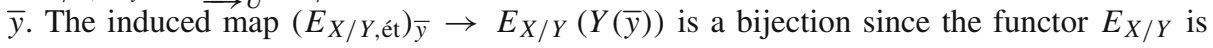
locally of finite presentation. The latter set equals $\left|X_{\bar{y}}\right| \cup\{\emptyset\}$ by Lemma 3.3 (ii).

Lemma 3.5 The sheaf $E_{X / Y}$ is locally constructible, i.e., for any scheme $T$ and morphism $\pi: T \rightarrow Y$, there is a natural isomorphism $\pi^{-1} E_{X / Y, e ́ t} \rightarrow E_{X \times{ }_{Y} T / T, e ́ t}$.

Proof There is a natural transformation $E_{X / Y \text {,ét }} \rightarrow \pi_{*} E_{X \times Y} T / T$,ét and hence by adjunction a natural transformation $\varphi: \pi^{-1} E_{X / Y \text {,ét }} \rightarrow E_{X \times Y} T / T$, ét. It is enough to verify that $\varphi$ is an isomorphism on geometric points. This follows from Lemma 3.4. 
Proposition 3.6 The sheaf $E_{X / Y}$ is an algebraic space and the natural morphism $e: E_{X / Y} \rightarrow Y$ is étale and representable.

Proof Indeed, this statement is equivalent to Lemma 3.5, cf. [1, Exp. IX, pf. Prop. 2.7] or [7, Ch. V, Thm. 1.5]. The space $E_{X / Y}$ is of finite presentation over $Y$ if and only if the sheaf $E_{X / Y}$ is constructible.

Remark 3.7 The algebraicity of $E_{X / Y}$ can also be shown as follows (and this is essentially the method used in the following section). The question is local on $Y$ so we can assume that $Y$ is affine and choose a diagram (*) as in the beginning of the introduction. It can then be shown that there is an étale representable and surjective morphism $E_{X^{\prime} / Y^{\prime}} \rightarrow E_{X / Y}$ and that $E_{X^{\prime} / Y^{\prime}}$ is represented by the scheme given as the gluing of two copies of $Y^{\prime}$ along $Y^{\prime} \backslash X^{\prime}$. Lemmas 3.2-3.5 are corollaries of this result and we do not need to use Appendix C.

The distinguished section of $E_{X / Y}(Y)$, corresponding to $W=\emptyset$, gives a section $j$ of $e: E_{X / Y} \rightarrow Y$. As the diagonal of $f: X \rightarrow Y$ is open, we have a morphism $i: X \rightarrow E_{X / Y}$ corresponding to the diagonal $\left\{X \rightarrow X \times_{Y} X\right\} \in E_{X / Y}(X)$.

Lemma 3.8 The morphism $i: X \rightarrow E_{X / Y}$ is a closed immersion and $E_{X / Y} \backslash i(X)=j(Y)$.

Proof Let $T$ be a $Y$-scheme and let $g: T \rightarrow E_{X / Y}$ be a morphism. To show that $i$ is a closed immersion, it is enough to show that the pull-back of $i$ along $g$ is a closed immersion. Let $w: W \rightarrow X \times{ }_{Y} T$ be the open immersion corresponding to $g$ so that $\pi_{2} \circ w: W \rightarrow T$ is a closed immersion. Then the squares
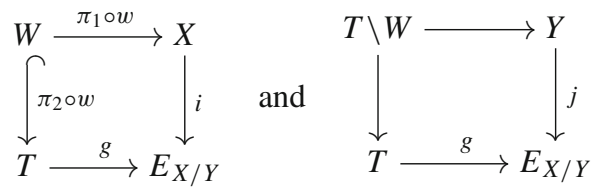

are commutative. The verification that these squares are cartesian is straight-forward.

Lemma 3.9 The triple e $: E_{X / Y} \rightarrow Y, i: X \rightarrow E_{X / Y}, j: Y \rightarrow E_{X / Y}$, is determined up to unique isomorphism by the condition that $E_{X / Y} \backslash i(X)=j(Y)$.

Proof Let $e^{\prime}: E^{\prime} \rightarrow Y, i^{\prime}: X \rightarrow E^{\prime}$ and $j^{\prime}: Y \rightarrow E^{\prime}$ be another triple of an étale morphism, a closed immersion and an open immersion such that $E^{\prime} \backslash i^{\prime}(X)=j^{\prime}(Y)$. There is only one possible morphism $\varphi: E^{\prime} \rightarrow E_{X / Y}$ such that $i=\varphi \circ i^{\prime}$ and $j=\varphi \circ j^{\prime}$, since the graph of $\varphi$ - an open subset of $E^{\prime} \times_{Y} E_{X / Y}$ - would be given as the union of the images of $\left(i^{\prime}, i\right): X \rightarrow E^{\prime} \times_{Y} E_{X / Y}$ and $\left(j^{\prime}, j\right): Y \rightarrow E^{\prime} \times_{Y} E_{X / Y}$.

The graph of the map $i^{\prime}$ determines an element of $E_{X / Y}\left(E^{\prime}\right)$, i.e., a morphism $\varphi: E^{\prime} \rightarrow$ $E_{X / Y}$, such that $i=\varphi \circ i^{\prime}$ and $j=\varphi \circ j^{\prime}$. As $\varphi$ is a bijective étale monomorphism, it is an isomorphism.

Proof (Theorem 1.2, representable case) We postpone the proof of the existence and uniqueness of $E_{X / Y}$ for non-representable morphisms $f: X \rightarrow Y$ to the following section. Similarly, for now, we only prove the functorial properties (viii) and (ix) in the representable case.

The existence of $e: E_{X / Y} \rightarrow Y, i$ and $j$ with the required properties, for an unramified morphism $f: X \rightarrow Y$ of algebraic spaces, follows from Proposition 3.6 and Lemma 3.8. The triple $(e, i, j)$ is unique with these properties by Lemma 3.9. That the triple commutes with base change follows from the uniqueness or directly from the functorial description. 
If $Y$ is an algebraic stack and $f: X \rightarrow Y$ is a representable unramified morphism, then we construct the representable and étale morphism $E_{X / Y} \rightarrow Y$ locally on $Y$ [6, Ch. 14]. We can also treat $E_{X / Y}$ as a cartesian lisse-étale sheaf of sets on $Y$.

This settles (i) and (ii) in the representable case. (iii) is trivial. (iv) If $E_{X / Y} \rightarrow Y$ is separated then $j$ is closed and $i$ is open and it follows that $f$ is étale and separated. If $f$ is étale then $E_{X / Y}=X \amalg Y$ and $E_{X / Y} \rightarrow Y$ is separated if and only if $f$ is separated. (v) That $E_{X / Y} \rightarrow Y$ is universally closed (resp. quasi-compact, resp. representable) if and only if $f$ is so, follows from the fact that $i$ is a closed immersion and that $i \amalg j$ is a surjective monomorphism (hence stabilizer preserving).

(vi) If $e: E_{X / Y} \rightarrow Y$ is quasi-separated then $j$ is quasi-compact so that $i$ is of constructible finite type by Proposition D.4. It follows that $f=e \circ i$ is quasi-separated and locally of constructible finite type. Conversely, if $f$ is quasi-separated and locally of constructible finite type, then so is $i$ by Proposition D.3. Hence $j$ is quasi-compact and, a fortiori, so is $i \amalg j: X \amalg Y \rightarrow E_{X / Y}$. As $f \amalg \operatorname{id}_{Y}=e \circ(i \amalg j)$ is quasi-separated it follows that $e: E_{X / Y} \rightarrow Y$ is quasi-separated. Finally, note that $e$ is finitely presented if and only if $e$ is quasi-compact and quasi-separated and that $f$ is of constructible finite type if and only if $f$ is quasi-compact, quasi-separated and locally of constructible finite type.

(viii) and (ix) (representable case) Let $g: V \rightarrow X$ be étale (resp. a closed immersion). We will construct a morphism $g_{*}: E_{V / Y} \rightarrow E_{X / Y}$ (resp. $g^{*}: E_{X / Y} \rightarrow E_{V / Y}$ ) using the functorial description.

In the étale case, an element of $E_{V / Y}(T)$ corresponding to an open subspace $W \subseteq V \times_{Y} T$ is mapped to the element corresponding to the composition $W \rightarrow V \times_{Y} T \rightarrow X \times{ }_{Y} T$. This composition, a priori only étale, is an open immersion since $W \rightarrow T$ is a closed immersion. That the pull-back of $i_{X}$ (resp. $j_{X}$ ) along $g_{*}$ is $i_{V}$ (resp. $j_{V}$ ) is easily verified. If $g$ is an open immersion, then $g_{*}$ is a monomorphism and hence an open immersion.

In the case of a closed immersion, an element of $E_{X / Y}(T)$ corresponding to an open subspace $W \subseteq X \times_{Y} T$ is mapped to the pull-back $g_{T}^{-1} W \subseteq V \times_{Y} T$. If $y: \operatorname{Spec}(k) \rightarrow Y$ is a point, then the morphism $g_{y}^{*}: E_{X_{y} / y}=X_{y} \cup\{y\} \rightarrow E_{V_{y} / y}=V_{y} \cup\{y\}$ is an isomorphism over the open and closed subscheme $V_{y} \cup\{y\}$ and maps $X_{y} \backslash V_{y}$ onto the distinguished point $y$. It follows that $i_{V}=g^{*} \circ i_{X} \circ g$, that $j_{V}=g^{*} \circ j_{X}$, that $g^{*}$ is surjective and that $g^{*}$ is a monomorphism if and only if $g$ is bijective.

(vii) If $e: E_{X / Y} \rightarrow Y$ is a local isomorphism, then $f=e \circ i$ is a local immersion. Conversely, assume that $f$ is a local immersion. The question whether $e$ is a local isomorphism is Zariski-local on $E_{X / Y}$ and $Y$. We can thus, using (viii), assume that $f$ is a closed immersion. Then $E_{X / Y}=Y \cup_{Y \backslash X} Y \rightarrow Y$ is a local isomorphism.

\section{The general case}

In this section we prove Theorem 1.2 for general unramified morphisms of stacks.

Definition 4.1 If $f: X \rightarrow Y$ is any (not necessarily representable) unramified morphism, then we define a stack $E_{X / Y}$ over $\mathbf{S c h}_{/ Y}$ (with the étale topology) as follows. The objects of the category $E_{X / Y}$ are 2-commutative diagrams

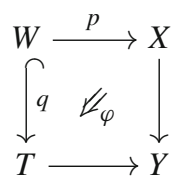


such that $T$ is a scheme, $(p, \varphi, q): W \rightarrow X \times_{Y} T$ is étale and $q$ is a closed immersion. Morphisms $\left(p^{\prime}, \varphi^{\prime}, q^{\prime}\right) \rightarrow(p, \varphi, q)$ are 2-commutative diagrams

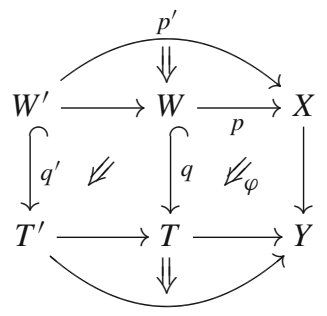

such that the left square is 2-cartesian and the pasting of the diagram is $\varphi^{\prime}$. The functor $E_{X / Y} \rightarrow \mathbf{S c h}_{/ Y}$ is the functor mapping the diagrams above onto their bottom rows. By étale descent, the category $E_{X / Y}$, which is fibered in groupoids, is a stack in the étale topology.

Lemma 4.2 Let $q: W \hookrightarrow T$ be a closed immersion and let $Z \rightarrow W$ be an étale morphism of stacks. Then $q_{*} Z \rightarrow T$ is étale. If $Z \rightarrow W$ is representable (resp. surjective, resp. an open immersion) then so is $q_{*} Z \rightarrow T$. Here $q_{*} Z$ denotes the stack over $\mathbf{S c h}_{/ T}$ which associates to a scheme $T^{\prime} \in \mathbf{S c h}_{/ T}$ the groupoid $\mathbf{H o m}_{W}\left(W \times_{T} T^{\prime}, Z\right)$.

Proof The question is fppf-local on $T$ and we can thus assume that $T$ is a scheme. Then $Z$ is Deligne-Mumford and we can pick an étale presentation $U \rightarrow Z$. It is enough to show that $q_{*} U \rightarrow q_{*} Z$ and $q_{*} U \rightarrow T$ are étale and representable and that the first map is surjective. We can thus assume that $Z \rightarrow W$ is representable. Then $Z$ is a locally constructible sheaf and it follows that $q_{*} Z$ is locally constructible by the proper base change theorem, i.e., $q_{*} Z \rightarrow T$ is étale and representable.

If $Z \rightarrow W$ is surjective, then so is $q_{*} Z \rightarrow T$. Indeed, this can be checked on stalks. Let $t \in T$ be a point. If $t \in W$, then $\left(q_{*} Z\right)_{\bar{t}}=Z_{\bar{t}} \neq \emptyset$. If $t \notin W$, then $\left(q_{*} Z\right)_{\bar{t}}=Z(\emptyset)$ is the final object - the one-point set.

If $Z \rightarrow W$ is an open immersion, then $q_{*} Z=T \backslash(W \backslash Z)$ as can be checked by passing to fibers.

Lemma 4.3 Let $g: V \rightarrow X$ be an étale morphism. Then there is a natural étale morphism $g_{*}: E_{V / Y} \rightarrow E_{X / Y}$. If $g$ is representable (resp. surjective, resp. an open immersion) then so is $g_{*}$.

Proof This is similar to the proof of Theorem 1.2 (viii) in the representable case. Let $\xi \in$ $E_{V / Y}$ be an object corresponding to morphisms $p: W \rightarrow V, q: W \hookrightarrow T$. We let $g_{*}(\xi) \in$ $E_{X / Y}$ be the object corresponding to $g \circ p$ and $q$. On morphisms $g_{*}$ is defined in the obvious way.

Let $T \rightarrow E_{X / Y}$ be a morphism corresponding to morphisms $p: W \rightarrow X$ and $q: W \hookrightarrow T$. If $T^{\prime}$ is a $T$-scheme, then the $T^{\prime}$-points of the pull-back $E_{V / Y} \times_{E_{X / Y}} T \rightarrow T$ is the groupoid of liftings of $p^{\prime}: W \times_{T} T^{\prime} \rightarrow X$ over $g: V \rightarrow X$, or equivalently, the groupoid of sections of $V \times_{X} W \times_{T} T^{\prime} \rightarrow W \times_{T} T^{\prime}$. This description is compatible with pull-backs so that $E_{V / Y} \times{ }_{E_{X / Y}} T$ is the stack $q_{*}\left(V \times_{X} W\right)$ which is algebraic and étale over $T$ by the previous lemma. Moreover, if $V \rightarrow X$ is representable (resp. surjective, resp. an open immersion) then so are $q_{*}\left(V \times_{X} W\right) \rightarrow T$ and $E_{V / Y} \rightarrow E_{X / Y}$.

Lemma 4.4 The stack $E_{X / Y}$ is algebraic. 
Proof Let $Y^{\prime} \rightarrow Y$ be a smooth presentation. Then $E_{X \times Y} Y^{\prime} / Y^{\prime} \rightarrow E_{X / Y}$ is representable, smooth and surjective. Replacing $X$ and $Y$ with $X \times_{Y} Y^{\prime}$ and $Y^{\prime}$ respectively, we can thus assume that $Y$ is a scheme.

Since $X \rightarrow Y$ is unramified, we have that $X$ is a Deligne-Mumford stack. Let $V \rightarrow X$ be an étale presentation. By Lemma 4.3, there is an étale representable surjection $E_{V / Y} \rightarrow E_{X / Y}$ and by Proposition 3.6, $E_{V / Y}$ is an algebraic space. This shows that $E_{X / Y}$ is algebraic.

Proof (Theorem 1.2, general case) We have already proved that $E_{X / Y}$ is algebraic in Lemma 4.4 and as in the representable case, we can define morphisms $i: X \rightarrow E_{X / Y}$ and $j: Y \rightarrow E_{X / Y}$. That $i$ is a closed immersion and $j$ is an open immersion such that $j(Y)$ is the complement of $i(X)$ follows exactly as in the proof of Lemma 3.8.

The uniqueness (which is up to unique 2-isomorphism) of $E_{X / Y}, i$ and $j$ satisfying $E_{X / Y} \backslash i(X)=j(Y)$ follows as in the proof of Lemma 3.9 (because any morphism $E \rightarrow E_{X / Y}$ commuting with $i$ and $j$ is representable).

(viii) is Lemma 4.3 and (ix) follows exactly as in the representable case.

\section{Applications}

There are two important consequences of Theorem 1.2. The first is that the classical description of unramified morphisms as étale-local embeddings remains valid when the target is not necessary Deligne-Mumford. The second is that we obtain a canonical factorization of an unramified morphism into a closed immersion and an étale morphism. The following example illustrates the first consequence.

Example 5.1 It can be shown that if $X \rightarrow Y$ is an étale, finitely presented and representable morphism or a closed immersion of stacks and $\widetilde{X} \rightarrow X$ is a blow-up, then there exists a blow-up $\widetilde{Y} \rightarrow Y$ and an $X$-morphism $\widetilde{Y} \times_{Y} X \rightarrow \widetilde{X}$. The analogous result for a representable unramified morphism $X \rightarrow Y$ of constructible finite type (e.g., of finite presentation) then follows from the existence of the étale envelope.

In the remainder of the section we outline an application where the canonicity of the étale envelope is crucial. It is shown in [12] that quasi-compact universally subtrusive morphisms (e.g., universally submersive morphisms between noetherian spaces) are morphisms of effective descent for the fibered category of finitely presented étale morphisms. Using Theorem 1.2 we obtain a similar effective descent statement for unramified morphisms.

Notation 5.2 Let $g: S^{\prime} \rightarrow S$ be a morphism of algebraic spaces. Let $S^{\prime \prime}=S^{\prime} \times{ }_{S} S^{\prime}$ be the fiber product and let $\pi_{1}, \pi_{2}: S^{\prime \prime} \rightarrow S^{\prime}$ be the two projections.

Proposition 5.3 (Descent) Let $g: S^{\prime} \rightarrow S$ be universally submersive. Let $X \rightarrow S$ and $Y \rightarrow S$ be unramified morphisms of algebraic spaces. Then the sequence

$$
\operatorname{Hom}_{S}\left(X_{\text {red }}, Y_{\text {red }}\right) \stackrel{g^{*}}{\longrightarrow} \operatorname{Hom}_{S^{\prime}}\left(X_{\text {red }}^{\prime}, Y_{\text {red }}^{\prime}\right) \stackrel{\pi_{1}^{*}}{\pi_{2}^{*}} \operatorname{Hom}_{S^{\prime \prime}}\left(X_{\text {red }}^{\prime \prime}, Y_{\text {red }}^{\prime \prime}\right)
$$

is exact. Here $X^{\prime}$ and $Y^{\prime}$ are the pull-backs of $X$ and $Y$ along $S^{\prime} \rightarrow S$, and $X^{\prime \prime}$ and $Y^{\prime \prime}$ are the pull-backs of $X$ and $Y$ along $S^{\prime \prime} \rightarrow S$.

Proof A morphism $f: X_{\text {red }} \rightarrow Y_{\text {red }}$ corresponds to an open subspace $\Gamma \subseteq X_{\text {red }} \times{ }_{S} Y_{\text {red }}$ such that the projection $\Gamma \rightarrow X_{\text {red }}$ is an isomorphism. Equivalently, since $Y \rightarrow S$ is unramified, an open subset $\Gamma \subseteq\left|X \times_{S} Y\right|$ corresponds to a morphism $X_{\text {red }} \rightarrow Y_{\text {red }}$ if and only if 
$\Gamma_{\text {red }} \rightarrow X_{\text {red }}$ is universally injective, surjective and proper. As $g$ is surjective, it follows that $\operatorname{Hom}_{S}\left(X_{\text {red }}, Y_{\text {red }}\right) \rightarrow \operatorname{Hom}_{S^{\prime}}\left(X_{\text {red }}^{\prime}, Y_{\text {red }}^{\prime}\right)$ is injective.

Now if $\Gamma^{\prime} \subseteq\left|X^{\prime} \times{ }_{S^{\prime}} Y^{\prime}\right|$ is an open subset such that $\pi_{1}^{-1} \Gamma^{\prime}=\pi_{2}^{-1} \Gamma^{\prime}$ as subsets of $\left|X^{\prime \prime} \times{ }_{S^{\prime \prime}} Y^{\prime \prime}\right|$, then $\Gamma^{\prime}$ is the pull-back of an open subset $\Gamma \subseteq\left|X \times_{S} Y\right|$ since $g$ is universally submersive. If in addition $\Gamma^{\prime}$ corresponds to a morphism $X_{\text {red }}^{\prime} \rightarrow Y_{\text {red }}^{\prime}$, then $\Gamma_{\text {red }}^{\prime} \rightarrow X_{\text {red }}^{\prime}$ is universally injective, surjective and proper. As $g$ is universally submersive, it follows that $\Gamma_{\text {red }} \rightarrow X_{\text {red }}$ also is universally injective, surjective and proper. Thus $\Gamma$ corresponds to a

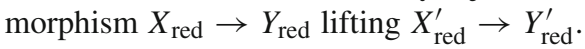

Theorem 5.4 (Effective descent) Let $g: S^{\prime} \rightarrow S$ be a quasi-compact and quasi-separated universally subtrusive morphism of algebraic spaces. Let $X^{\prime} \rightarrow S^{\prime}$ be an unramified morphism of constructible finite type (e.g., of finite presentation) of algebraic spaces equipped with a "reduced descent datum" relative to $S^{\prime} \rightarrow S$, i.e., an isomorphism $\theta:\left(\pi_{1}^{*} X^{\prime}\right)_{\mathrm{red}} \rightarrow$

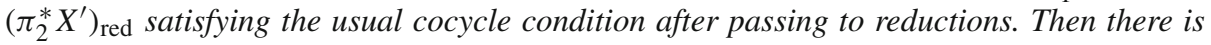
a unique unramified morphism $X \rightarrow S$ of constructible finite type and a schematically dominant morphism $X^{\prime} \rightarrow X$ such that $X^{\prime} \rightarrow X \times{ }_{S} S^{\prime}$ is a nil-immersion.

Proof Let $X_{i}^{\prime \prime}=\pi_{i}^{*} X^{\prime}$ for $i=1,2$ so that $X^{\prime \prime}:=\left(X_{1}^{\prime \prime}\right)_{\text {red }} \cong\left(X_{2}^{\prime \prime}\right)_{\text {red. }}$. Consider the étale envelopes $E_{X^{\prime} / S^{\prime}}, E_{X^{\prime \prime} / S^{\prime \prime}}$ and $E_{X_{i}^{\prime \prime} / S^{\prime \prime}}$. The nil-immersions $X^{\prime \prime} \hookrightarrow X_{i}^{\prime \prime}$ induce natural isomorphisms $E_{X_{i}^{\prime \prime} / S^{\prime \prime}} \rightarrow E_{X^{\prime \prime} / S^{\prime \prime}}$. As the étale envelope commutes with pull-back, there is a canonical isomorphism $E_{X^{\prime \prime} / S^{\prime \prime}} \cong \pi_{1}^{*} E_{X^{\prime} / S^{\prime}} \cong \pi_{2}^{*} E_{X^{\prime} / S^{\prime}}$ which equips $E_{X^{\prime} / S^{\prime}}$ with a descent datum.

The morphism $E_{X^{\prime} / S^{\prime}} \rightarrow S^{\prime}$ is étale and of finite presentation. Thus, it descends to a morphism $E \rightarrow S$ which is étale and of finite presentation [12, Thm. 5.17]. The induced morphism $h: E_{X^{\prime} / S^{\prime}} \rightarrow E$ is a pull-back of $g$ and thus universally subtrusive. As $h$ is surjective and $\pi_{1}^{-1}\left(i^{\prime}\left(X^{\prime}\right)\right)=\pi_{2}^{-1}\left(i^{\prime}\left(X^{\prime}\right)\right)$ as sets, there is a unique subset $X \subseteq E$ such that $h^{-1}(X)=i^{\prime}\left(X^{\prime}\right)$. Since $h$ is subtrusive and $i^{\prime}\left(X^{\prime}\right) \subseteq E_{X^{\prime} / S^{\prime}}$ is closed and constructible, it follows that $X$ is closed and constructible. We consider the set $X$ as a closed subspace of $E$ by taking the "schematic image" of $X^{\prime} \hookrightarrow E_{X^{\prime} / S^{\prime}} \rightarrow E$. Then $X \rightarrow S$ satisfies the conditions of the theorem.

Corollary 5.5 Let $\mathbf{U n r}_{\text {cons }}(S)$ be the category of unramified morphisms $X \rightarrow S$ of constructible finite type such that $X$ is reduced and let $\mathbf{U n r}_{\text {cons }}\left(S^{\prime} \rightarrow S\right)$ be the category of unramified morphisms $X^{\prime} \rightarrow S^{\prime}$, of constructible finite type, equipped with a reduced descent datum and with $X^{\prime}$ reduced. There is a natural functor $\mathbf{U n r}_{\text {cons }}(S) \rightarrow \mathbf{U n r}_{\text {cons }}\left(S^{\prime} \rightarrow S\right)$ taking $X \rightarrow S$ to $\left(X \times{ }_{S} S^{\prime}\right)_{\mathrm{red}} \rightarrow S^{\prime}$ and the induced descent datum. This functor is an equivalence of categories.

\section{Appendix A: Algebraic spaces and stacks}

A sheaf of sets $F$ on the category of schemes Sch with the étale topology is an algebraic space if there exists a scheme $X$ and a morphism $X \rightarrow F$ which is represented by surjective étale morphisms of schemes [11, Déf. 5.7.1], i.e., for any scheme $T$ and morphism $T \rightarrow F$, the fiber product $X \times_{F} T$ is a scheme and $X \times_{F} T \rightarrow T$ is surjective and étale.

A stack is a category fibered in groupoids over Sch with the étale topology satisfying the usual sheaf condition [6]. A morphism $f: X \rightarrow Y$ of stacks is representable if for any scheme $T$ and morphism $T \rightarrow Y$, the 2-fiber product $X \times_{Y} T$ is an algebraic space. A stack $X$ is algebraic if there exists a smooth presentation, i.e., a smooth, surjective and 
representable morphism $U \rightarrow X$ where $U$ is a scheme. A stack $X$ is Deligne-Mumford if there exists an étale presentation. A stack $X$ is Deligne-Mumford if and only if $X$ is algebraic and the diagonal $\Delta_{X}$ is unramified. A morphism $f: X \rightarrow Y$ of stacks is quasi-separated if the diagonal $\Delta_{X / Y}$ is quasi-compact and quasi-separated, i.e., if both $\Delta_{X / Y}$ and its diagonal are quasi-compact.

Remark A.1 (Quasi-separatedness) We do not require that algebraic spaces and stacks are quasi-separated nor that the diagonal of an algebraic stack is separated. The queasy reader may assume that the diagonals of all stacks and algebraic spaces are separated and quasi-compact (as in [5,6]) but this is not necessary in this paper. The reader should however note that unless we work with noetherian stacks or finitely presented unramified morphisms, stacks and algebraic spaces with non-quasi-compact diagonals will appear.

The diagonal of a (not necessarily quasi-separated) algebraic space is representable by schemes. This follows by effective fppf-descent of monomorphisms which are locally of finite type. Indeed, more generally the class of locally quasi-finite and separated morphisms is an effective class in the fppf-topology (cf. [8, App.], [2, Exp. X, Lem. 5.4] or [11, pf. of 5.7.2])

The diagonal of an algebraic stack $X$ is representable. This follows by [6, pf. of Prop. 4.3.1] as [6, Cor. 1.6.3] generalizes to arbitrary algebraic spaces.

The characterization of Deligne-Mumford stacks as algebraic stacks with unramified diagonal is valid for arbitrary algebraic stacks. Indeed, the proof of [6, Thm. 8.1] does not use that the diagonal is separated and quasi-compact.

\section{Appendix B: Unramified and étale morphisms of stacks}

We use the modern terminology of unramified morphisms [10]: an unramified morphism of schemes is a formally unramified morphism which is locally of finite type (and not necessarily locally of finite presentation). Equivalently, an unramified morphism is a morphism locally of finite type such that the diagonal is an open immersion [3, 17.4.1.2]. Recall that an étale morphism of schemes is a formally étale morphism which is locally of finite presentation or equivalently, a flat and unramified morphism which is locally of finite presentation $[3,17.6 .2]$. These definitions generalize to include non-representable morphisms as follows:

Definition B.1 A morphism $f: X \rightarrow Y$ of algebraic stacks is unramified if $f$ is locally of finite type and the diagonal $\Delta_{f}$ is étale. A morphism $f: X \rightarrow Y$ of algebraic stacks is étale if $f$ is locally of finite presentation, flat and unramified.

For representable $f$ this definition of unramified agrees with the usual since an étale monomorphism is an open immersion [3, Thm. 17.9.1]. The notions of unramified and étale are fpqc-local on the target and étale-local on the source [3, 2.2.11 (iv), 2.7.1, 17.7.3, 17.7.7].

Proposition B.2 Let $f: X \rightarrow Y$ be a morphism of algebraic stacks. The following are equivalent:

(i) $f$ is étale.

(ii) $f$ is smooth and unramified.

Proof As a smooth morphism is flat and locally of finite presentation (ii) implies (i). To see that (i) implies (ii), take a smooth presentation $U \rightarrow X$. If $f$ is étale then $U \times_{X} U \rightarrow U \times_{Y} U$ 
is étale. Thus, the projections $U \times{ }_{Y} U \rightarrow U$ are smooth at the points in the image of $U \times{ }_{X} U$. Since $U \times{ }_{X} U \rightarrow U$ is surjective and $U \rightarrow Y$ is flat, it follows that $U \rightarrow Y$ is smooth by flat descent and, a fortiori, that $X \rightarrow Y$ is smooth.

Proposition B.3 Let $f: X \rightarrow Y$ be a morphism of algebraic stacks. The following are equivalent:

(i) $f$ is unramified.

(ii) $f$ is locally of finite type and for every point $\operatorname{Spec}(k) \rightarrow Y$ we have that $X \times_{Y}$ $\operatorname{Spec}(k) \rightarrow \operatorname{Spec}(k)$ is unramified.

(iii) $f$ is locally of finite type and for every point $\operatorname{Spec}(k) \rightarrow Y$ we have that $X \times_{Y} \operatorname{Spec}(k)$ is geometrically reduced, Deligne-Mumford and discrete.

Proof Clearly (i) $\Longrightarrow$ (ii). If $f$ is representable, then it is well-known that (ii) $\Longrightarrow$ (iii) $\Longrightarrow$ (i) $[3,17.4 .1 .2]$. For general $f$, to see that (ii) $\Longrightarrow$ (iii) we can assume that $Y=\operatorname{Spec}(k)$ so that $X$ is Deligne-Mumford. As both (ii) and (iii) are étale-local on $X$ we can also assume that $X$ is a scheme so that $f$ is representable and (ii) $\Longrightarrow$ (iii) by the representable case.

If (iii) holds, then the fibers of the diagonal are unramified and hence $\Delta_{f}$ is unramified, i.e., $f$ is Deligne-Mumford. Let $Y^{\prime} \rightarrow Y$ be a smooth presentation and let $X^{\prime} \rightarrow X \times_{Y} Y^{\prime}$ be an étale presentation. Then the representable morphism $X^{\prime} \rightarrow Y^{\prime}$ also satisfies condition (iii) and hence is unramified. This shows that (iii) $\Longrightarrow$ (i).

In the remainder of this section we will show that the definitions of unramified and étale given above have a more standard formal description.

Definition B.4 Let $S$ be a stack and let $X$ and $Y$ be stacks over $S$. We let $\operatorname{Hom}_{S}(X, Y)$ be the groupoid with objects 2-commutative diagrams

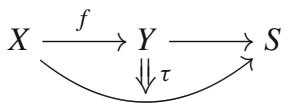

and morphisms $\varphi:\left(f_{1}, \tau_{1}\right) \rightarrow\left(f_{2}, \tau_{2}\right), 2$-commutative diagrams

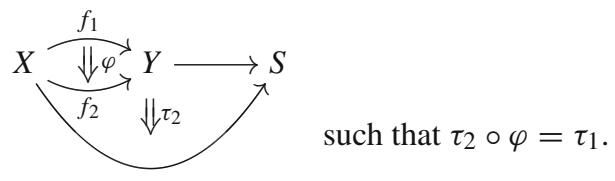

We note that if $Y \rightarrow S$ is representable, then the groupoid $\operatorname{Hom}_{S}(X, Y)$ is equivalent to a set.

Definition B.5 Let $f: X \rightarrow Y$ be a morphism of stacks. We say that $f$ is formally unramified (resp. formally Deligne-Mumford, resp. formally smooth, resp. formally étale) if for every $Y$-scheme $T$ and every closed subscheme $T_{0} \hookrightarrow T$ defined by a nilpotent ideal sheaf the functor

$$
\operatorname{Hom}_{Y}(T, X) \rightarrow \operatorname{Hom}_{Y}\left(T_{0}, X\right)
$$

is fully faithful (resp. faithful, resp. essentially surjective, resp. an equivalence of categories). 
Remark B.6 The functor $\operatorname{Hom}_{Y}(T, X) \rightarrow \operatorname{Hom}_{Y}\left(T_{0}, X\right)$ is essentially surjective if and only if for every 2-commutative diagram

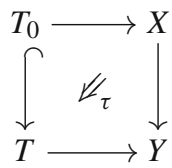

there exists a morphism $T \rightarrow X$ and a 2-commutative diagram

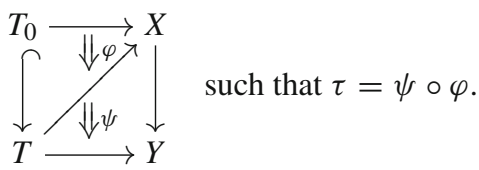

If $f: X \rightarrow Y$ is locally of finite presentation, then it can be shown that it is enough to consider strictly henselian $T$ and closed subschemes $T_{0} \hookrightarrow T$ defined by a square-zero ideal, cf. [6, Prop. 4.15 (ii)].

Formally unramified (resp. ...) morphisms are stable under base change, products and composition, cf. [3, Prop. 17.1.3].

Proposition B.7 Let $f: X \rightarrow Y$ be a morphism of stacks. Then $f$ is formally unramified (resp. formally Deligne-Mumford) if and only if the diagonal $\Delta_{f}$ is formally étale (resp. formally unramified).

Proof Let $T$ be a $Y$-scheme and let $j: T_{0} \hookrightarrow T$ be a closed subscheme defined by a nilpotent ideal. Let $\left(f_{1}, \tau_{1}\right)$ and $\left(f_{2}, \tau_{2}\right)$ be two objects of $\operatorname{Hom}_{Y}(T, X)$. This determines a morphism $F=\left(f_{1}, \tau_{2}^{-1} \circ \tau_{1}, f_{2}\right): T \rightarrow X \times_{Y} X$. Conversely, a morphism $F: T \rightarrow$ $X \times_{Y} X$ gives rise to a (non-unique) pair $\left(f_{1}, \tau_{1}\right),\left(f_{2}, \tau_{2}\right)$ of objects in $\operatorname{Hom}_{Y}(T, X)$ such that $F=\left(f_{1}, \tau_{2}^{-1} \circ \tau_{1}, f_{2}\right)$.

Fix a pair of objects $\left(f_{1}, \tau_{1}\right),\left(f_{2}, \tau_{2}\right)$ and a morphism $F: T \rightarrow X \times_{Y} X$ as above. As the diagonal of $f$ is representable, the groupoid $\operatorname{Hom}_{X \times_{Y} X}(T, X)$ is equivalent to the set $\operatorname{Hom}_{X \times Y} X(T, X):=\pi_{0} \operatorname{Hom}_{X \times_{Y} X}(T, X)$. There is a natural bijection between the set of 2-morphisms $\operatorname{Hom}\left(f_{1}, f_{2}\right)$ and the set $\operatorname{Hom}_{X \times_{Y} X}(T, X)$. Thus $\operatorname{Hom}\left(f_{1}, f_{2}\right) \rightarrow \operatorname{Hom}\left(f_{1} \circ\right.$ $j, f_{2} \circ j$ ) is bijective (resp. injective) if and only if $\operatorname{Hom}_{X \times_{Y} X}(T, X) \rightarrow \operatorname{Hom}_{X \times_{Y} X}\left(T_{0}, X\right)$ is bijective (resp. injective).

Corollary B.8 Let $f: X \rightarrow Y$ and $g: Y \rightarrow Z$ be two morphisms.

(i) If $g \circ f$ is formally Deligne-Mumford then so is $f$.

(ii) If $g \circ f$ is formally unramified and $g$ is formally Deligne-Mumford, then $f$ is formally unramified.

Corollary B.9 Let $f: X \rightarrow Y$ be a morphism of stacks.

(i) $f$ is smooth if and only if $f$ is locally of finite presentation and formally smooth.

(ii) $f$ is étale if and only if $f$ is locally of finite presentation and formally étale.

(iii) $f$ is unramified if and only if $f$ is locally of finite type and formally unramified.

(iv) $f$ is Deligne-Mumford (i.e., $\Delta_{f}$ is unramified) if and only if $f$ is formally DeligneMumford. 
Proof If $f$ is representable, then (i), (ii) and (iii) are definitions and (iv) is trivial. For general $f$, statement (iii) [resp. (iv)] follows from Proposition B.7 using statement (ii) [resp. (iii)] for the representable diagonal $\Delta_{f}$. Statement (i) is [6, Prop. 4.15 (ii)]. Finally (ii) follows from Proposition B.2 and (i) and (iii).

\section{Appendix C: Auxiliary limit results}

In this appendix we give some fairly standard limit results. For simplicity we state these results for algebraic spaces although they remain valid for algebraic stacks.

Proposition C.1 Let $S_{0}$ be an algebraic space and let $S=\lim _{\lambda} S_{\lambda}$ be an inverse limit of algebraic spaces that are affine over $S_{0}$. Let $X$ be a quasi-compact and quasi-separated algebraic space and let $X \rightarrow S$ be a morphism locally of finite presentation. Then there exists an index $\lambda$, a quasi-compact and quasi-separated algebraic space $X_{\lambda}$, a morphism $X_{\lambda} \rightarrow S_{\lambda}$ locally of finite presentation and an $S$-isomorphism $X_{\lambda} \times S_{\lambda} S \rightarrow X$. If $X \rightarrow S$ is étale then it can be arranged so that $X_{\lambda} \rightarrow S_{\lambda}$ also is étale.

Proof Since $X$ is quasi-compact, we can assume that $S_{0}$ is quasi-compact after replacing $S_{0}$ by an open subspace. Let $V_{0} \rightarrow S_{0}$ be an étale presentation with $V_{0}$ an affine scheme. Let $V_{\lambda}=V_{0} \times S_{0} S_{\lambda}$ and $V=V_{0} \times S_{0} S$. Finally choose an affine scheme $U$ and an étale morphism $U \rightarrow V \times{ }_{S} X$ such that $U \rightarrow X$ is surjective. Note that $U \rightarrow X$ and $U \rightarrow V$ are of finite presentation. Let $R=U \times{ }_{X} U$ and note that $j: R \rightarrow U \times{ }_{S} U$ is a monomorphism of finite presentation as $X$ is quasi-separated.

Since $U \rightarrow V$ and $j$ are of finite presentation, there is for sufficiently large $\lambda$ a finitely presented scheme $U_{\lambda} \rightarrow V_{\lambda}$, a finitely presented monomorphism $j_{\lambda}: R_{\lambda} \rightarrow U_{\lambda} \times{ }_{S_{\lambda}} U_{\lambda}$ and cartesian diagrams
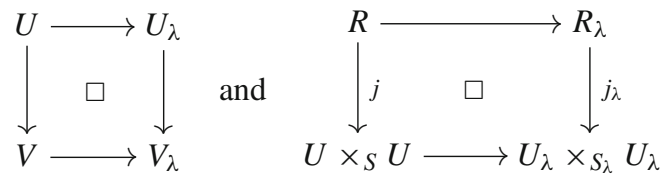

such that $s_{\lambda}, t_{\lambda}: R_{\lambda} \rightarrow U_{\lambda}$ are étale with $s_{\lambda}=\pi_{1} \circ j_{\lambda}$ and $t_{\lambda}=\pi_{2} \circ j_{\lambda}$, and $R_{\lambda}$ is quasicompact. The morphism $j_{\lambda}=\left(s_{\lambda}, t_{\lambda}\right)$ defines an equivalence relation if and only if

(R) the pull-back of $j_{\lambda}$ along $\Delta_{U_{\lambda}}: U_{\lambda} \rightarrow U_{\lambda} \times{ }_{S_{\lambda}} U_{\lambda}$ is an isomorphism,

(S) the pull-back of $j_{\lambda}$ along $\left(t_{\lambda}, s_{\lambda}\right): R_{\lambda} \rightarrow U_{\lambda} \times{ }_{S_{\lambda}} U_{\lambda}$ is an isomorphism, and

(T) the pull-back of $j_{\lambda}$ along $\left(s \circ \pi_{1}, t \circ \pi_{2}\right): R_{\lambda} \times{ }_{t_{\lambda}, U_{\lambda}, s_{\lambda}} R_{\lambda} \rightarrow U_{\lambda} \times S_{\lambda} U_{\lambda}$ is an isomorphism.

The pull-back of the above maps along $U \rightarrow U_{\lambda}, R \rightarrow R_{\lambda}$ and $R \times{ }_{U} R \rightarrow R_{\lambda} \times U_{\lambda} R_{\lambda}$ respectively are isomorphisms since $j$ is an equivalence relation. Noting that $j_{\lambda}$ is of finite presentation and $U_{\lambda}, R_{\lambda}$ and $R_{\lambda} \times_{U_{\lambda}} R_{\lambda}$ are quasi-compact, we conclude that $j_{\lambda}$ is an equivalence relation for sufficiently large $\lambda$ by [3, Thm. 8.10 .5 (i)]. The quotient $X_{\lambda}$ of this equivalence relation is a quasi-compact and quasi-separated algebraic space which is locally of finite presentation over $S_{\lambda}$. The last assertion follows from [3, Prop. 17.7.8 (ii)].

Note that Proposition C.1 reduces to the standard limit result on finitely presented objects if $S_{0}$ is quasi-compact and quasi-separated. 
Proposition C.2 Let $S_{0}$ be an affine scheme and let $S=\lim _{\lambda} S_{\lambda}$ be an inverse limit of affine $S_{0}$-schemes. Let $X_{0}$ be an algebraic space and let $f_{0}: X_{0} \stackrel{\leftarrow}{\rightarrow} S_{0}$ be of finite type and quasiseparated. Let $f_{\lambda}: X_{\lambda} \rightarrow S_{\lambda}$ and $f: X \rightarrow S$ denote the base changes of $f_{0}$. Then $f$ is a monomorphism (resp. closed immersion) if and only if $f_{\lambda}$ is a monomorphism (resp. closed immersion) for sufficiently large $\lambda$.

Proof The condition is clearly sufficient. To see that the condition is necessary for the property "monomorphism", recall that a morphism $f$ is a monomorphism if and only if its diagonal $\Delta_{f}$ is an isomorphism. As the diagonal is strongly representable and finitely presented the necessity in this case follows from [3, Thm. 8.10 .5 (i)]. If $f$ is a closed immersion then by the previous case $f_{\lambda}$ is a monomorphism for sufficiently large $\lambda$. In particular $f_{\lambda}$ is quasifinite and separated so that $f_{\lambda}$ is strongly representable [6, Thm. A.2] and Zariski's main theorem [3, Cor. 18.12.13] gives rise to a factorization $X_{\lambda} \rightarrow Y_{\lambda} \rightarrow S_{\lambda}$ of $f_{\lambda}$ where the first morphism is a quasi-compact open immersion and the second morphism is finite. As $X \rightarrow Y_{\lambda} \times S_{\lambda} S$ is an open and closed immersion so is $X_{\lambda} \rightarrow Y_{\lambda}$ for sufficiently large $\lambda$. In particular $X_{\lambda} \rightarrow S_{\lambda}$ is a proper monomorphism and hence a closed immersion.

More generally Proposition C.2 holds for properties such as: proper, finite, affine, quasiaffine, separated; but not for other properties such as being an isomorphism.

\section{Appendix D: Morphisms of constructible finite type}

In this section we define morphisms (locally) of constructible finite type. A morphism (locally) of finite presentation is (locally) of constructible finite type and a morphism (locally) of constructible finite type is (locally) of finite type. For morphisms of noetherian stacks, all these notions coincide.

Let $X$ be a scheme. Recall that a subset $W \subseteq X$ is ind-constructible (resp. pro-constructible) if locally $W$ is a union (resp. an intersection) of constructible subsets [4, Déf. 7.2.2]. If $p: U \rightarrow X$ is locally of finite presentation and surjective, then $W$ is ind-constructible (resp. pro-constructible, resp. constructible) if and only if $p^{-1}(W)$ is so [4, Cor. 7.2.10]. Now let $X$ be an algebraic stack. We define a subset $W \subseteq X$ to be ind-constructible (resp. pro-constructible, resp. constructible) if $p^{-1}(W)$ is so for some presentation $p: U \rightarrow X$ with $U$ a scheme. This definition does not depend on the choice of presentation.

Definition D.1 Let $f: X \rightarrow Y$ be a morphism of algebraic stacks. The morphism $f$ is indconstructible if the image under $f$ of any ind-constructible subset is ind-constructible. If this holds after arbitrary base change $Y^{\prime} \rightarrow Y$, then we say that $f$ is universally ind-constructible.

The primary example of an ind-constructible morphism is a morphism which is locally of finite presentation [4, Prop. 7.2.3].

Definition D.2 A morphism $f: X \rightarrow Y$ of stacks is locally of constructible finite type if $f$ is locally of finite type and universally ind-constructible. A morphism $f$ is of constructible finite type if $f$ is quasi-compact, quasi-separated and locally of constructible finite type.

Morphisms (locally) of finite presentation are (locally) of constructible finite type. The image of a pro-constructible set under a quasi-compact morphism is pro-constructible [4, Prop. 7.2.3]. It follows that a morphism of constructible finite type takes constructible subsets to constructible subsets [4, Prop. 7.2.9].

Proposition D.3 Let $f: X \rightarrow Y$ and $g: Y \rightarrow Z$ be morphisms of algebraic stacks. 
(i) If $f$ and $g$ are locally of constructible finite type, then so is $g \circ f$.

(ii) If $g \circ f$ is locally of constructible finite type and if $g$ is locally of finite type, then $f$ is locally of constructible finite type.

Proof (i) is obvious. (ii) As the diagonal of $g$ is locally of finite presentation, we have that $f$ is the composition of a morphism locally of constructible finite type and a morphism locally of finite presentation, hence locally of constructible finite type.

Proposition D.4 Let $f: Z \rightarrow X$ be a closed immersion of algebraic stacks. The following are equivalent:

(i) $f$ is of constructible finite type.

(ii) The subset $|Z| \subseteq|X|$ is constructible.

(iii) The open immersion $X \backslash Z \rightarrow X$ is quasi-compact.

Proof Immediate from the fact that an open immersion is pro-constructible if and only if it is quasi-compact [4, Prop. 7.2.3].

Not every quasi-separated morphism of finite type is of constructible finite type. For example, there are closed immersions which are not constructible. A morphism locally of finite presentation, e.g., an étale morphism, is of constructible finite type if and only if it is of finite presentation.

Let $f: X \rightarrow Y$ be an unramified morphism with a factorization $X \hookrightarrow X_{1} \rightarrow Y$ where $X \hookrightarrow X_{1}$ is a nil-immersion and $X_{1} \rightarrow Y$ is unramified and of finite presentation. Then $f$ is of constructible finite type. Conversely, if $Y$ is quasi-compact and quasi-separated it is likely that every unramified morphism $f$ of constructible finite type has such a factorization.

Open Access This article is distributed under the terms of the Creative Commons Attribution Noncommercial License which permits any noncommercial use, distribution, and reproduction in any medium, provided the original author(s) and source are credited.

\section{References}

1. Artin, M., Grothendieck, A., Verdier, J.L. (eds.): Théorie des topos et cohomologie étale des schémas. Séminaire de Géométrie Algébrique du Bois Marie 1963-1964 (SGA 4). Dirigé par M., Artin, A., Grothendieck J.L. Verdier. Avec la collaboration de P. Deligne et B. Saint-Donat, Lecture Notes in Mathematics, vol. 269, 270, 305. Springer, Berlin (1972-1973)

2. Demazure, M., Grothendieck, A. (eds.): Schémas en groupes. Séminaire de Géométrie Algébrique du Bois Marie 1962/64 (SGA 3). Dirigé par M. Demazure et A. Grothendieck. Lecture Notes in Mathematics, vol. 151-153. Springer-Verlag, Berlin (1970)

3. Grothendieck, A.: Éléments de géométrie algébrique. IV. Étude locale des schémas et des morphismes de schémas, Inst. Hautes Études Sci. Publ. Math. (1964-1967), nos. 20, 24, 28, 32

4. Grothendieck, A.: Éléments de géométrie algébrique. I. Le langage des schémas, 2nd edn, Die Grundlehren der mathematischen Wissenschaften in Einzeldarstellungen, vol. 166. Springer, Berlin (1971)

5. Knutson, D.: Algebraic spaces. Lecture Notes in Mathematics, vol. 203. Springer, Berlin (1971)

6. Laumon, G., Moret-Bailly, L.: Champs algébriques, Ergebnisse der Mathematik und ihrer Grenzgebiete. 3. Folge. A Series of Modern Surveys in Mathematics. Springer, Berlin (2000)

7. Milne, J.S.: Étale cohomology, Princeton Mathematical Series, vol. 33. Princeton University Press, Princeton (1980)

8. Murre, J.P.: Representation of unramified functors. Applications (according to unpublished results of A. Grothendieck), Séminaire Bourbaki, t. 17, 1964/1965, Exp. No. 294, Secrétariat mathématique, Paris, p. 19 (1966)

9. Mustaţă, A.M., Mustaţă, A.: The structure of a local embedding and Chern classes of weighted blow-ups, Preprint, (2009). http://arXiv.org/abs/0812.3101v2 
10. Raynaud, M.: Anneaux locaux henséliens, Lecture Notes in Mathematics, vol. 169. Springer, Berlin (1970)

11. Raynaud, M., Gruson, L.: Critères de platitude et de projectivité: Techniques de "platification" d'un module. Invent. Math. 13, 1-89 (1971)

12. Rydh, D.: Submersions and effective descent of étale morphisms, Bull. Soc. Math. France 138(2), 211-260 (2010). http://arXiv.org/abs/0710.2488v3 